\title{
A Wideband Correlation and Detection Module Based on Substrate-Integrated Waveguide Technology for Radio Astronomy Applications
}

\author{
Juan Luis Cano ${ }^{\circledR}$, Enrique Villa, Angel Mediavilla, and Eduardo Artal, Member, IEEE
}

\begin{abstract}
A wideband $(30 \%$ relative bandwidth) correlation and detection module based on substrate-integrated waveguide (SIW) technology intended for a radio astronomy polarimeter is presented. The SIW circuit is a six-port network with two input ports that are correlated in two hybrid couplers and their corresponding output signals are routed to Schottky diode detectors, which are designed using microstrip technology and assembled within the same system. The designed SIW structure includes hybrid couplers, power dividers, a $90^{\circ}$ phase shifter, and $90^{\circ}$ bends, providing a real implementation of a functional system with improved bandwidth performance from 35 to 47 GHz. Experimental results are in concordance with simulations, and they validate the module operation for the proposed application.
\end{abstract}

Index Terms-Correlation, polarimeter, radio astronomy, radiometer, substrate-integrated waveguide (SIW), wideband.

\section{INTRODUCTION}

$\mathbf{S}$ UBSTRATE-INTEGRATED waveguide (SIW) is a relatively new technology that is having an increasing interest since it was proposed by Deslandes and Wu [1]. The main reason for its success is the combination of the advantages of planar technology, such as lightness, compactness, and reduced manufacture cost, with those of waveguide technology, such as low insertion loss and higher quality factors.

On the other hand, radio astronomy is among the most demanding applications because a slight improvement in the receiver sensitivity has a huge impact on the observing time and the scientific knowledge itself. In particular, the study of the cosmic microwave background (CMB), both in intensity and in polarization, pushes the technology to its limits, requiring state-of-the-art subsystems, due to the faintness of the received signals.

The most critical components affecting the sensitivity of a radiometer or polarimeter are the first subsystems in the receiver chain. A high insertion loss degrades the equivalent noise temperature, and therefore these elements, such as feedhorns, polarizers, and orthomode transducers (OMT), are

Manuscript received September 28, 2017; revised January 11, 2018; accepted March 16, 2018. This work was supported by the Spanish Ministry of Economy and Competitiveness under Project AYA2013-49759-EXP. (Corresponding author: Juan Luis Cano.)

The authors are with the Departamento de Ingeniería de Comunicaciones, Universidad de Cantabria, 39005 Santander, Spain (e-mail: juanluis.cano@unican.es).

Color versions of one or more of the figures in this paper are available online at http://ieeexplore.iee.org.

Digital Object Identifier 10.1109/TMTT.2018.2823305 designed using the waveguide technology. They are normally cooled to cryogenic temperatures to reduce even more its noise contribution. However, not only low losses are an important guideline for these kinds of receivers but also the number of receivers (pixels) that can be accommodated in the instrument has a significant influence in the overall performance. For this reason, modern radio astronomy receivers are multipixel cameras, where the increasing number of pixels is the current trend. In this context, the SIW technology has a clear opportunity for the development of light, compact, highly integrated, and relatively cheap back ends.

The QUIJOTE project [2], [3] is a multipixel radio astronomy ground-based experiment with the aim of characterizing the CMB polarization, among other scientific goals, through the measurement of the $Q, U$, and $I$ Stokes parameters simultaneously from 10 to $47 \mathrm{GHz}$ in different subbands. The calculation of these Stokes parameters is carried out through the correlation and detection of the incoming signals. These operations are performed in a specific unit in each pixel called correlation and detection module (CDM).

Although the final election for the design of the CDM in the QUIJOTE project was the waveguide technology, this paper presents the development of a full system implementing the correlation, detection, and level accommodation functionalities in Q-band (35-47 GHz, 30\% instantaneous bandwidth) with most of the RF circuitry implemented in the SIW technology. Once correlated, the signals are detected using Schottky diode detectors implemented in the planar technology and integrated in the same module. Finally, the system incorporates adjustable video amplifiers to accommodate the detected voltages to suitable values for the subsequent data acquisition electronics unit. The proposed CDM scheme demonstrates the feasibility of the SIW technology to perform complex operations, beyond its individual parts, in highly demanding applications such as radio astronomy.

Section II presents the pixel scheme and the CDM block diagram in order to explain its principle of operation. Section III provides detailed information about the CDM design focusing on each subsystem separately. CDM measurement results are presented in Section IV, whereas the conclusion is presented in Section V.

\section{PRinciple OF OPERATION}

The polarimeter scheme, before the CDM, selected for each pixel in the QUIJOTE project (30- and $40-\mathrm{GHz}$ bands) is 


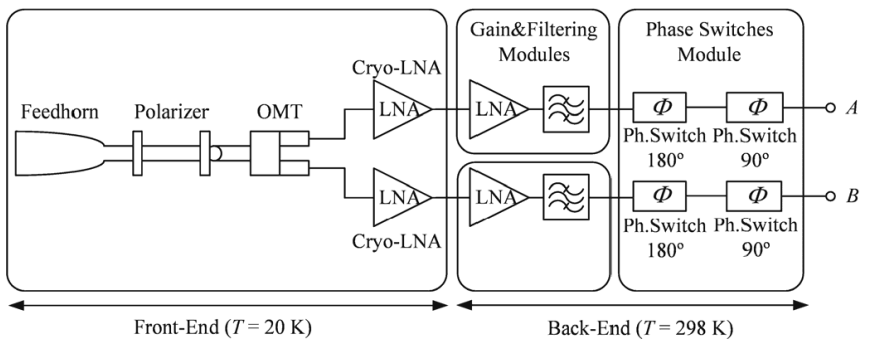

(a)

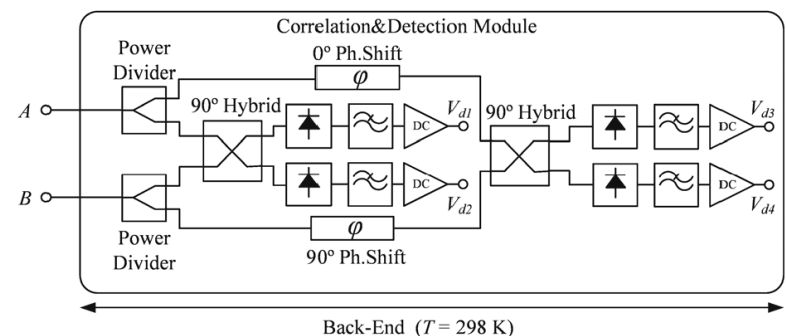

(b)

Fig. 1. Polarimeter scheme for every pixel in the QUIJOTE project. (a) Frontend components, at cryogenic temperature, are followed by an amplification stage and a phase-switching module. (b) CDM correlates, detects, and amplifies its input signals.

presented in Fig. 1(a). The output signals in Fig. 1(a) would be the input signals to the CDM designed using the SIW technology and shown in Fig. 1(b).

According to the known astrophysics data, the CMB radiation signal that enters the system is a faint noise-like signal with a tiny linearly polarized component (anisotropies of the order of a few tens of microkelvin [4]). This component, together with its fluctuations, is of great interest for astrophysicists since it provides a great insight into the universe origin and evolution.

At the OMT output ports, there are two noise-like signals proportional to the left-hand and right-hand circular components of the incoming signal. These two signals are amplified using first cryogenic very low-noise amplifiers (LNAs) and then room temperature LNAs. After band definition through suitable bandpass filters, these signals can be phase modulated using a fast switching technique in the corresponding module [5]. With a $180^{\circ}$ and a $90^{\circ}$ phase switch in each branch, a total number of 16 phase states can be accomplished. (Four different relative phase differences between branches repeated four times each.) This redundancy provides a way to eliminate some systematic errors at the postprocessing stage, and the switching speed contributes to avoid the problems produced by the $1 / f$ noise.

The $I, Q$, and $U$ Stokes parameters that enable to characterize the intensity and the linear polarization of the incoming signal can be calculated with the following equations:

$$
\begin{aligned}
I & =|A|^{2}+|B|^{2} \\
Q & =2 \operatorname{Re}\left(A^{*} \cdot B\right) \\
U & =-2 \operatorname{Im}\left(A^{*} \cdot B\right)
\end{aligned}
$$

where $A$ and $B$ are the CDM input signals [see Fig. 1(b)]. From (1)-(3), a correlation between the input signals is

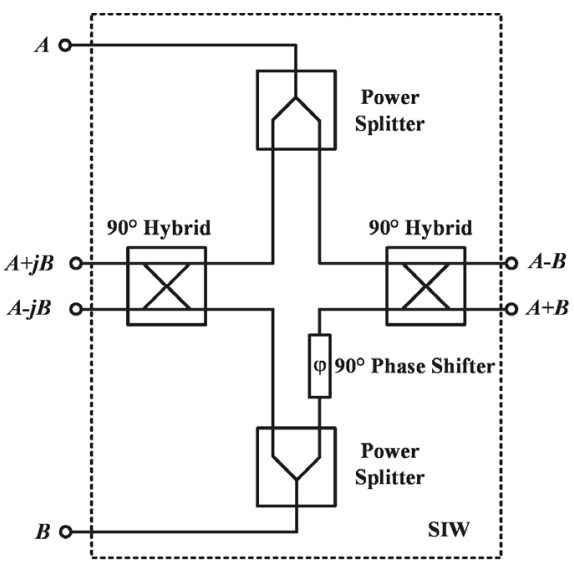

Fig. 2. Block diagram of the subsystems integrated in the same substrate using the SIW technology.

required. This correlation is carried out in broadband hybrid couplers. For obtaining real and imaginary parts, a $90^{\circ}$ phase shifter is necessary in one branch [see Fig. 1(b)]. Finally, the correlated signals are detected with Schottky diode detectors, and the dc output voltage components are amplified. The Stokes parameters are obtained through linear combinations of the detected voltages $\left(V_{d 1}-V_{d 4}\right)$. The specific operations with these output signals for each parameter depend on the phase state of the phase switches module.

\section{Correlation And Detection Module Design}

The CDM is a six-port unit with two broadband highfrequency inputs $(A$ and $B)$ and four dc outputs $\left(V_{d 1}-V_{d 4}\right)$. All the high-frequency subsystems are integrated in the same substrate using the SIW technology, as shown schematically in Fig. 2. This scheme is the classical configuration of a six-port junction that has been already demonstrated in the SIW technology [6], [7] and evolved technologies [8], [9]. However, these works dealt with limited relative bandwidths of less than $20 \%$ since they were aimed for demonstration purposes or selective radar applications. Only in [8], a similar application to the one intended in this paper was presented, obtaining good results in $20 \%$ bandwidth. Thus, these developments avoided the practical frequency limitations that arise for some subsystems in the SIW technology when bandwidths of $30 \%$ or wider are chased, as it is the case of this application where the bandwidth has a direct relationship with the instrument sensitivity.

The CDM input connections are standard-flange WR22 ports which are coupled to the SIW substrate using wideband WR22-to-SIW direct transitions [11]. From this point on, the design of each subsystem covering the $35-47-\mathrm{GHz}$ band in the SIW technology is detailed in Sections II-A-II-E.

\section{A. $90^{\circ}$ Hybrid Coupler}

The coupler is designed first in the standard waveguide technology following the Riblet-modified configuration of [12] for the Q-band (waveguide dimensions: $a=5.7 \mathrm{~mm}$ 


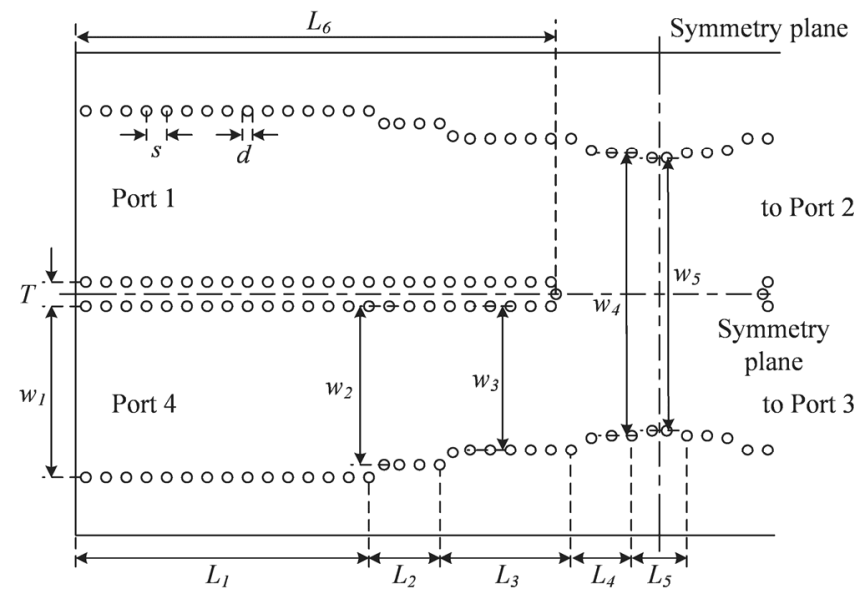

Fig. 3. Coupler designed in the SIW technology and dimensions definition. The drawing has symmetry planes; therefore, the right-hand side has been deleted for clearance.

TABLE I

HyBRID COUPLER FinAl DIMENSIONS

\begin{tabular}{cccc}
\hline \hline \multirow{2}{*}{ Parameter } & Value $(\mathrm{mm})$ & Parameter & Value $(\mathrm{mm})$ \\
\hline$s$ & 0.4 & $w_{5}$ & 5.4 \\
\hline$d$ & 0.2 & $L_{1}$ & 5.8 \\
\hline$T$ & 0.48 & $L_{2}$ & 1.4 \\
\hline$w_{1}$ & 3.4 & $L_{3}$ & 2.6 \\
\hline$w_{2}$ & 3.15 & $L_{4}$ & 1.2 \\
\hline$w_{3}$ & 2.85 & $L_{5}$ & 1.09 \\
\hline$w_{4}$ & 5.6 & $L_{6}$ & 9.5 \\
\hline \hline
\end{tabular}

and $b=2.85 \mathrm{~mm})$. In this coupler, the signal entering the structure, for example, at Port 1 in Fig. 3 is directly transmitted to Port 2 and coupled to Port 3, with equal power magnitudes, using different $\mathrm{H}$-plane matching steps to obtain the desired bandwidth performance. Port 4 is the isolated port, and a very small fraction of the signal reaches this port. Theoretically, for a symmetric $3-\mathrm{dB}$ coupler, the isolation performance should be exactly equal to the input return loss. The obtained structure dimensions are scaled to take into account the dielectric constant of the substrate that is used for the SIW circuits. In this case, the substrate is RO3003 from Rogers Corporation $\left(\varepsilon_{r}=3, h=0.254 \mathrm{~mm}, \tan \delta=0.0017\right.$ at $50 \mathrm{GHz}$, and metallization thickness $t=17 \mu \mathrm{m}$ ). Finally, the metallic lateral walls are implemented using metallized holes with diameter $d$ and longitudinal spacing $s$ that form the classical SIW structure. These parameters are selected conveniently to avoid undesired radiation between holes [13]; thus, $d=0.2 \mathrm{~mm}$ and $s=0.4 \mathrm{~mm}$. Applying the equations from [14], different widths, $w_{i}$, in the coupler can be calculated (see Fig. 3). The obtained dimensions for the coupler are presented in Table I.

The hybrid coupler circuit was simulated with a full3-D electromagnetic tool such as CST Microwave Studio. The simulation results are presented in Fig. 4 showing an input return loss and isolation around $25 \mathrm{~dB}$ and a transmission loss of $3.45 \pm 0.3 \mathrm{~dB}$ in the whole design band $(35-47 \mathrm{GHz})$. The phase difference between output ports is $90.1^{\circ} \pm 0.5^{\circ}$ in the band.

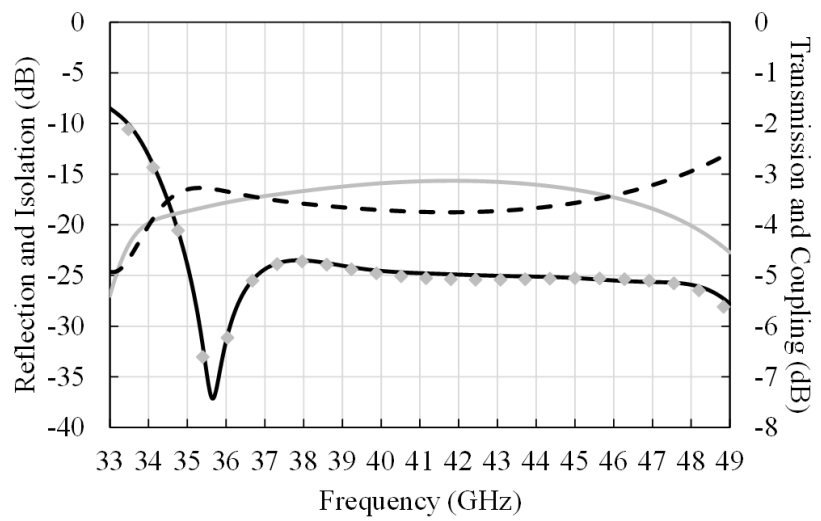

Fig. 4. Simulated parameters of the designed hybrid coupler. Black solid line: reflection. Black dashed line: transmission. Gray solid line: coupling. Gray diamonds: isolation.

\section{B. $90^{\circ}$ Phase Shifter}

Probably, $90^{\circ}$ phase shifter is the most challenging subsystem in the CDM since it has to show a nearly constant phase difference between the reference line and the delay line over a wide bandwidth, a good input matching, and a low insertion loss to not imbalance the hybrid couplers, and it is advisable to keep equal length between both lines to maintain symmetry in the structure, which improves its isolation performance.

Traditional techniques for waveguide phase shifting such as different line lengths [15] or lines widths [16] are frequency dependent, and therefore, they are narrowband in nature. Since the frequency dependence on these two structures has opposite behaviors, both techniques can be combined resulting in an improved phase shifter with wider bandwidth [17]. However, this phase shifter and its reference line show different physical lengths, which becomes more significant for large phase shifts, hindering its integration and not being suitable for the intended application.

The best option to accomplish all the requirements is to include a single dielectric slab centered in the waveguide. This dielectric slab may have a higher or a lower dielectric constant than the SIW substrate, and it may be implemented in a continuous way or using holes [18], [19]. In order to facilitate its manufacture, in our design, a low dielectric constant (air) slab was selected and, instead of using a sequence of increasing/decreasing-diameter holes [18], a continuous increasing/decreasing-width air-filled slab was implemented due to the large phase shift required (see Fig. 5).

Parameters $w_{1}, s$, and $d$ are obtained from the hybrid coupler design, whereas parameters $w_{\mathrm{in}}, w_{m}$, and $L_{\mathrm{sb}}$ are optimized for obtaining the best performance in terms of matching and phase difference. The simulation process, carried out with CST, resulted in the following parameter values: $w_{\text {in }}=0.1 \mathrm{~mm}, w_{m}=0.4 \mathrm{~mm}$, and $L_{\mathrm{sb}}=15.9 \mathrm{~mm}$. In Fig. 6, the simulated performance, both reflection and transmission, for the shift line is presented, whereas the phase difference between this line and its reference is shown in Fig. 7. Return loss is better than $25 \mathrm{~dB}$ in the analyzed frequency range, and the insertion loss is around $0.2 \mathrm{~dB}$ in the band of interest. 


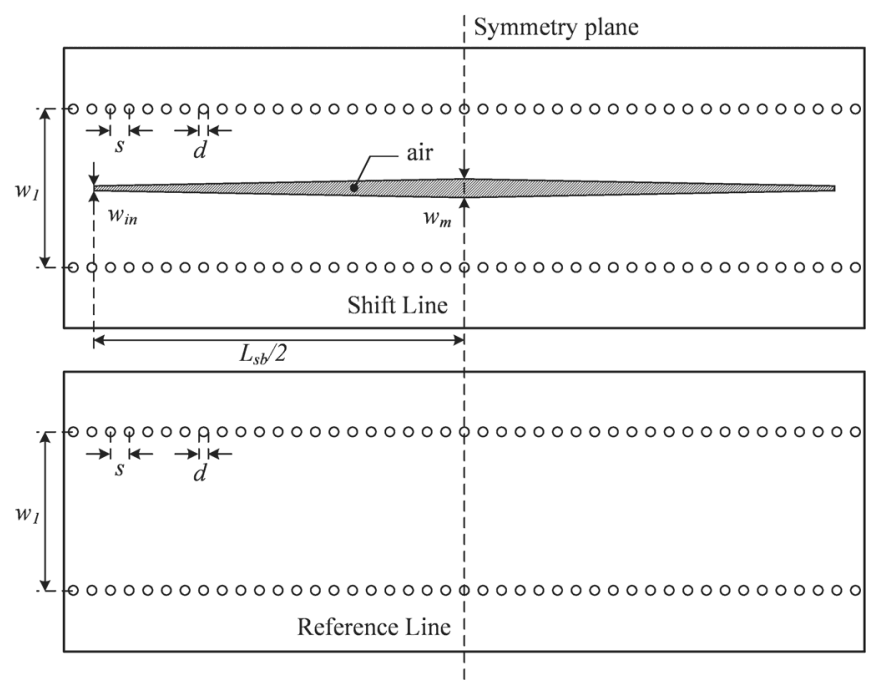

Fig. 5. $90^{\circ}$ phase shifter designed in the SIW technology and dimensions definition.

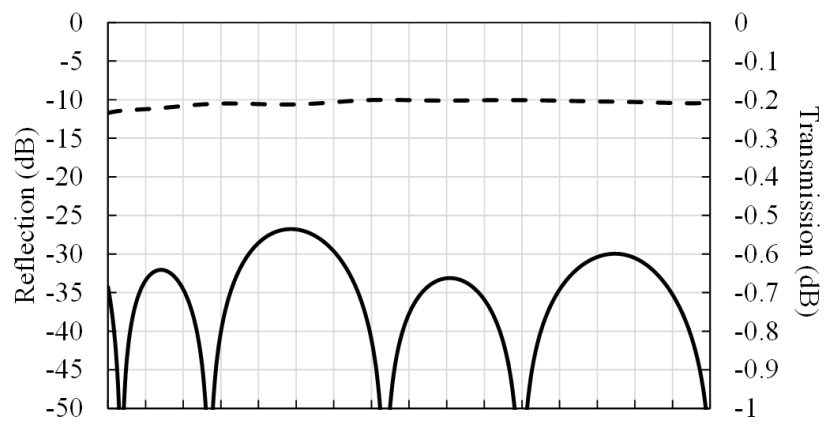

3334353637383940414243444546474849

Frequency $(\mathrm{GHz})$

Fig. 6. Simulated parameters of the shift line in the designed phase shifter Black solid line: reflection. Black dashed line: transmission.

This last value is similar to the simulated insertion loss of the reference line.

\section{3-dB Power Divider}

In-phase equal power dividers are necessary at the input of the module structure in order to obtain adequate samples to perform the correlation operations. From a functionality point of view, hybrid couplers could be used to carry out this operation (output signals would be swapped between the correlation hybrid couplers), but the isolated ports of these couplers for power division would need to be conveniently loaded, which represents an additional drawback.

Effective T-junction or Y-junction power dividers have been demonstrated in the SIW technology with an inductive metallic post or a straight metallic wall, respectively [20]. These structures are direct translations from their waveguide counterparts which have been used for decades. For this reason, traditional techniques, such as the inclusion of different matching steps in the common port [21], can be applied to these SIW structures in order to obtain broader bandwidths.

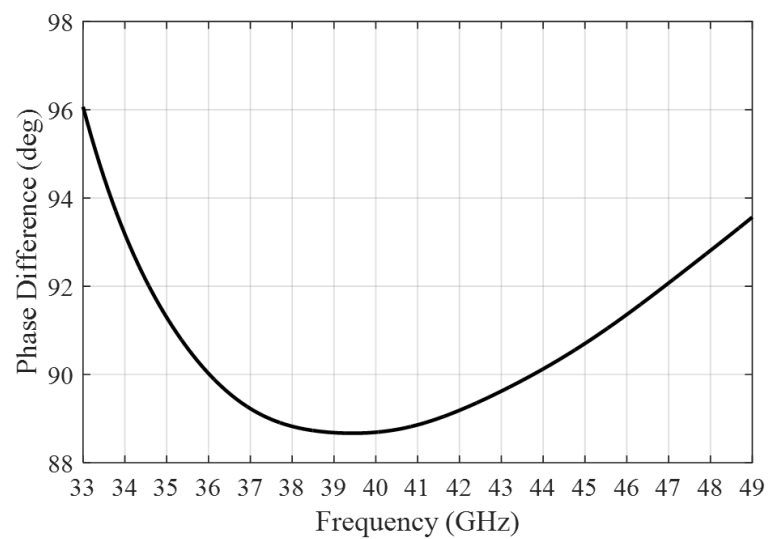

Fig. 7. Simulated phase difference between the shift line and the reference line.

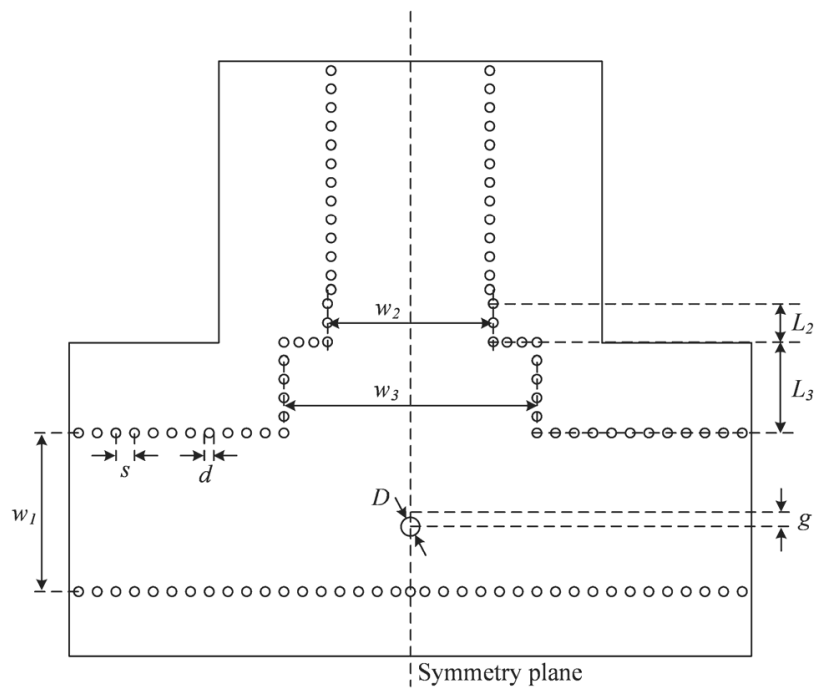

Fig. 8. T-junction power divider designed in the SIW technology and dimensions definition.

The power divider designed for the CDM uses an inductive metallic post at the T-junction together with two matching steps $\left(w_{2}, L_{2}\right.$ and $\left.w_{3}, L_{3}\right)$ at the common port to obtain a good reflection in the whole frequency range of interest. This metallic post has a diameter $D$ and is drilled with an offset $g$ regarding the junction center. The parameters that define the SIW structure $\left(s, d\right.$, and $\left.w_{1}\right)$ are the same as in the previous circuits. A drawing of the designed power divider with the parameters definition is presented in Fig. 8, whereas the simulated performance obtained with CST is shown in Fig. 9. Final values of design parameters are given in Table II.

\section{D. $90^{\circ}$ Bends}

The interconnection of the previously designed subcircuits in the SIW substrate requires several $90^{\circ}$ bends (see Fig. 2). Due to the number of bends, apart from a broadband performance, these components need to approach a nearly transparent behavior from an electrical point of view, i.e., their return loss must be kept above $40 \mathrm{~dB}$, and their insertion loss need to be minimized. 


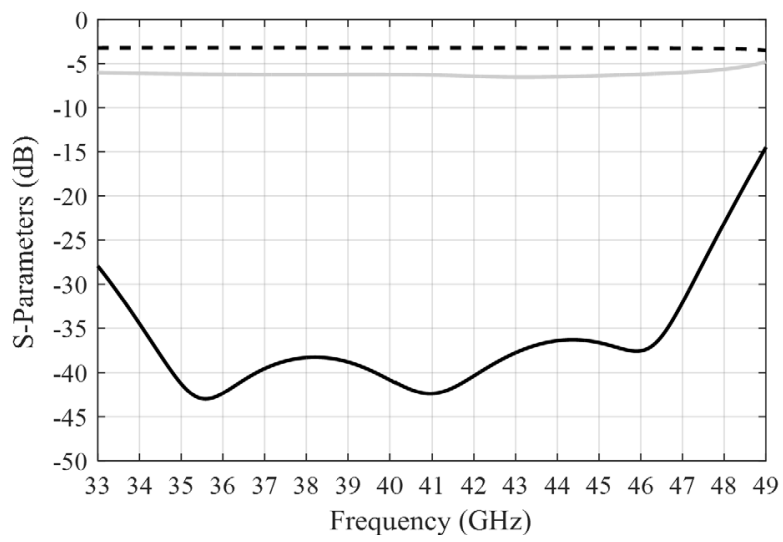

Fig. 9. Simulated performance of the 3-dB power divider. Black solid line: reflection at common port. Black dashed line: transmission from common port to one output port. Gray solid line: transmission between output ports.

TABLE II

POWER Divider FinAL Dimensions

\begin{tabular}{cc}
\hline \hline Parameter & Value $(\mathrm{mm})$ \\
\hline$w_{2}$ & 3.55 \\
\hline$w_{3}$ & 5.42 \\
\hline$L_{2}$ & 0.82 \\
\hline$L_{3}$ & 1.95 \\
\hline$g$ & 0.32 \\
\hline$D$ & 0.4 \\
\hline \hline
\end{tabular}

Different options such as rounded, mitered, multistepped, or multimitered corners have been proposed for accomplishing arbitrary angle bends in the waveguide technology fulfilling the required performances [22]. Among these options, the simplest ones have been demonstrated in the SIW technology showing electrical behaviors that suit our application [23]. Following this idea, for the CDM, a rounded $90^{\circ}$ bend has been designed with parameter $R$ as the only design parameter (see Fig. 10). The angular offset between holes in the rounded wall, $\theta$, is not optimized, but it must be maintained small in order to avoid signal leakage. Therefore, this parameter was fixed to $\theta=5^{\circ}$, and the bend radius was optimized with CST for the desired performance, obtaining a value of $R=3.81 \mathrm{~mm}$. The other parameters $\left(s, d\right.$, and $\left.w_{1}\right)$ have the same values than in previous circuits. The simulation results of this bend are shown in Fig. 11.

\section{E. Module Integration}

All the subcircuits are integrated together, and the resulting SIW substrate is inserted in a module machined from an aluminum block. This module includes the WR22-to-SIW direct transitions [11], space for the microwave detectors, and a cavity at the back to assemble a PCB containing four video amplifiers with adjustable gain in differential configuration. A top-view picture of the module fully assembled, with the cover removed, is shown in Fig. 12, whereas a close view of the SIW board is presented in Fig. 13.

The microwave detectors are based on the HSCH-9161 Schottky diode from Keysight Technologies, whose impedance

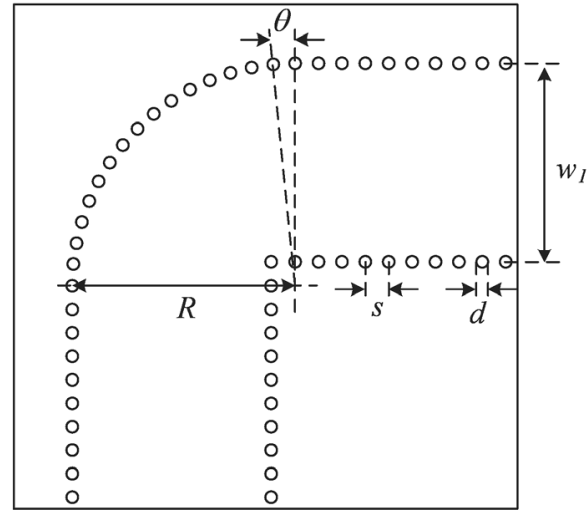

Fig. 10. $90^{\circ}$ bend designed in the SIW technology and parameter definition.

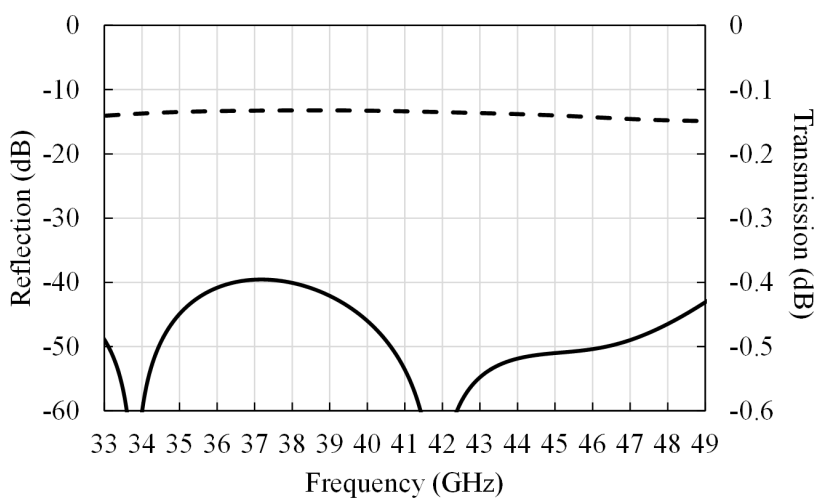

Fig. 11. Simulated performance of $90^{\circ}$ bend. Black solid line: reflection. Black dashed line: transmission.

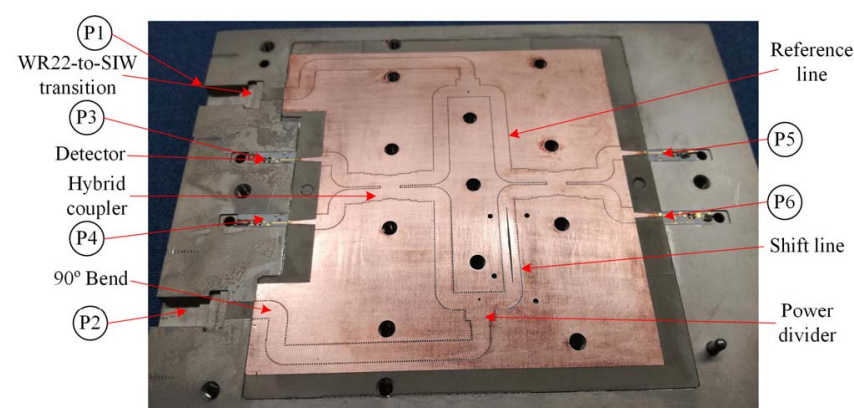

Fig. 12. Picture of the assembled CDM designed in the SIW technology. Ports are numbered from $\mathrm{P}_{1}$ to $\mathrm{P}_{6}$.

is matched to $50 \Omega$ using thin-film resistors and microstrip lines in a circuit designed on alumina substrate $\left(\varepsilon_{r}=9.9\right.$ and $h=0.254 \mathrm{~mm}$ ). Information about a previous version of this detector designed for a lower band can be found in [24] and [25]. In order to match the detector circuit to the SIW output ports, a taper-like microstrip-to-SIW transition [1] was implemented.

\section{Characterization And Measurement Results}

The characterization of the CDM performance under the proposed integration is limited due to the presence of the microwave detectors, which provide a dc output voltage, and 


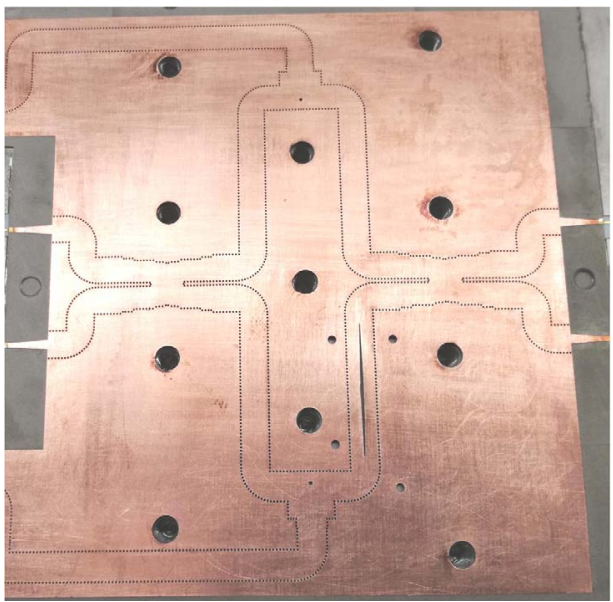

Fig. 13. Detailed photography of the SIW board.

therefore, most of the S-parameters of the SIW structure cannot be obtained.

The strategy followed to measure the CDM behavior is twofold: first, the input ports return loss and the isolation between them can be characterized with a VNA connected to this module, and second, the RF-to-dc conversion can be measured introducing known RF input signals varying their frequency and recording the obtained output voltages.

As stated before, the RF input ports return loss and isolation are measured connecting the CDM input ports to the VNA ports. The reflections and the transmission between these ports are shown in Fig. 14(a), where return loss values around $15-20 \mathrm{~dB}$ and an isolation better than $30 \mathrm{~dB}$ can be appreciated. These results are in concordance with the simulated data presented in Fig. 14(b), obtained from the full structure (WR22-to-SIW transitions included) introduced in CST Microwave Studio. For estimation purposes, the simulated transmission magnitudes from both input ports to the same output port are included in Fig. 14(b). These insertion losses include the 3-dB division of the power splitter and the $3-\mathrm{dB}$ division in a hybrid coupler; therefore, the estimated losses are around 2.5-3 dB for the whole SIW structure. An effective way of reducing these insertion losses would be to increase the substrate thickness [26]. Regarding the ports return loss and isolation, the measured and simulation results agree well with those reported for similar structures in [6]-[10] but showing an improved bandwidth. Only the simulated transmission coefficients seem to be slightly higher than those presented in the literature, which could be attributed to the larger number of bends or longer access lines of the proposed structure.

For the RF-to-dc conversion characterization, the two input signals of the CDM must be carefully controlled both in amplitude and phase in order to obtain meaningful results. A single frequency tone is generated using a signal generator model 83650B from Hewlett-Packard with a constant output power, and a frequency swept from 33 to $49 \mathrm{GHz}$. This signal is conducted through a coaxial cable and introduced in a broadband waveguide hybrid coupler obtaining two similar

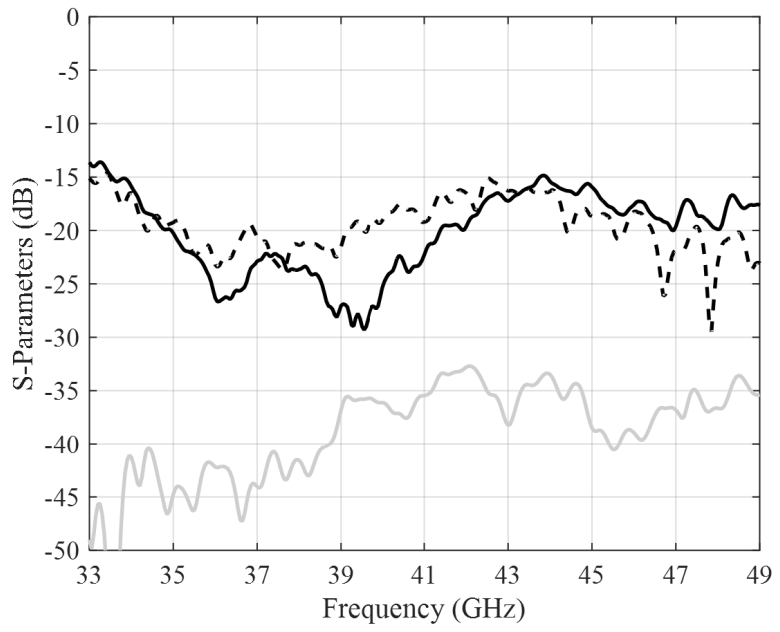

(a)

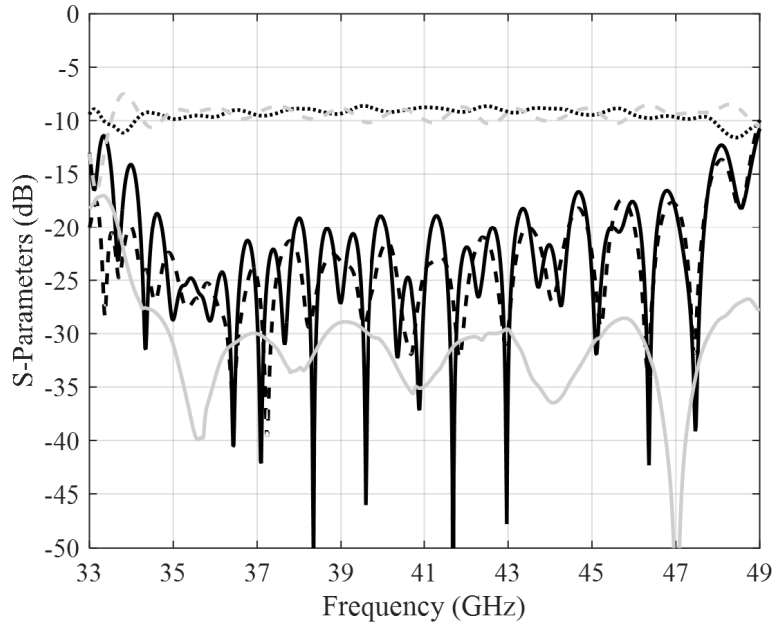

(b)

Fig. 14. (a) Measured RF performance of the CDM. Black solid line: reflection at $\mathrm{P}_{1}$. Black dashed line: reflection at $\mathrm{P}_{2}$. Gray solid line: transmission between input ports. (b) Simulated RF performance of the CDM. Black solid line: reflection at $\mathrm{P}_{1}$. Black dashed line reflection at $\mathrm{P}_{2}$. Gray solid line: transmission between input ports. Gray dashed line: transmission from $\mathrm{P}_{1}$ to $\mathrm{P}_{3}$. Black dotted line: transmission from $\mathrm{P}_{2}$ to $\mathrm{P}_{3}$.

signals with the same power and $90^{\circ}$ out of phase. These two tones are the input signals of the CDM. According to this configuration and the block diagram from Fig. 1(b), the theoretical expected output signals would be $V_{d 1}=0$, $V_{d 2}=A$, and $V_{d 3}=V_{d 4}=A / 2$ across the band, being $A$ the output dc voltage corresponding to the detected sum of both input signals. The output voltages are measured with a datalogger and stored for data processing. Fig. 15 shows the measured voltages across the band.

Analyzing these measured curves and considering the nonidealities of the manufactured circuits, some conclusions can be indicated: ripples are produced for mismatch between subcircuits, $V_{d 3}$ and $V_{d 4}$ are not equal due to the phase error in the $90^{\circ}$ phase shifter, and $V_{d 1}$ is not zero because of a small amplitude imbalance between branches. Despite this, the obtained results can be regarded as adequate if we consider this as a broadband application, and the integrated values across 


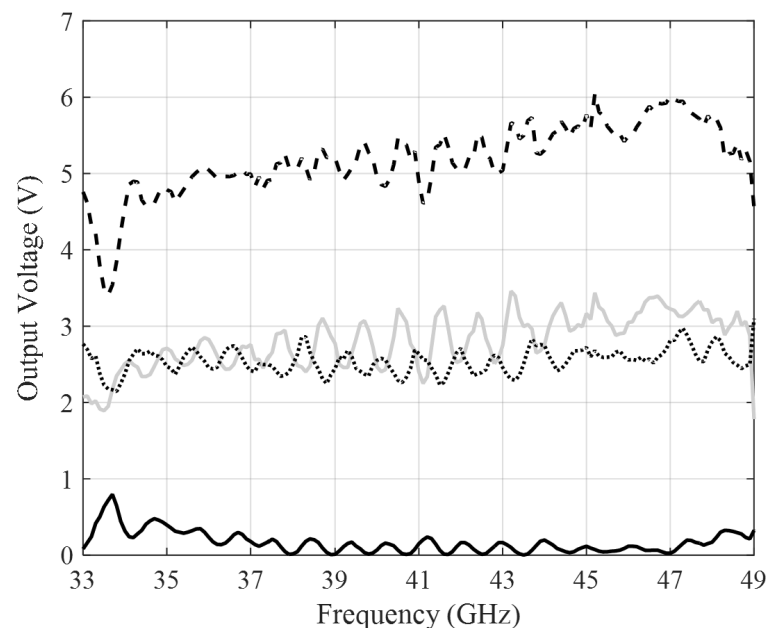

Fig. 15. Measured output voltages, according to labels in Fig. 1(b), for the designed CDM. Black solid line: $V_{d 1}$. Black dashed line: $V_{d 2}$. Gray solid line: $V_{d 3}$. Black dotted line: $V_{d 4}$.

the band are finally stored under real operation; the whole receiver requires a comprehensive calibration.

In addition, with the measured results presented in Fig. 15, a tentative value of isolation between Stokes parameters $U$ and $I$ can be obtained. In the proposed test setup, the values $I=Q=V_{d 2}-V_{d 1}$ and $U=V_{d 3}-V_{d 4}=0$ are theoretically expected. However, and due to nonidealities, $I>Q$ and $U>$ 0 , leading to an isolation of around $I \operatorname{sol}_{U / I}=13.3 \mathrm{~dB}$. Although the last value is insufficient to detect the $\mathrm{CMB}$ polarization by itself, it can be greatly improved through the calibration process that has to be carried out on the whole receiver prior to instrument operation. For example, redundant phase states (four phase states repeated four times each) are switched in each switching cycle in order to obtain mean values or fitting curves and to avoid some systematic errors. Moreover, the phase imbalance of the two signals entering the CDM is planned to be adjusted to compensate and minimize the difference $V_{d 3}-V_{d 4}$ which directly improves the isolation parameter. Therefore, these results provide a good starting point and validate the proposed module based on the SIW technology.

\section{CONClusion}

A system performing the correlation, detection, and level accommodation functionalities that incorporates a six-port network implemented in the SIW technology with improved bandwidth performance has been designed, implemented, and demonstrated. This system enables to obtain the Stokes parameters $(I, Q$, and $U)$ in a Q-band polarimeter for radio astronomy applications. The SIW substrate, which includes all the required subsystems, is assembled in a dedicated aluminum module together with power detectors that provide the detected voltages $\left(V_{d 1}-V_{d 4}\right)$, and video amplifiers that produce adequate voltage levels for the subsequent data acquisition equipment.

Measured results are in concordance with the simulations and they validate not only the module scheme but also its performance for the proposed application, representing a clear alternative for back-end modules based on the SIW technology in modern multipixel cameras for radio astronomy.

\section{ACKNOWLEDGMENT}

The authors would like to thank A. R. Perez and E. M. Cuerno, for their contribution to circuits manufacture and assembly.

\section{REFERENCES}

[1] D. Deslandes and K. Wu, "Integrated microstrip and rectangular waveguide in planar form," IEEE Microw. Wireless Compon. Lett., vol. 11, no. 2, pp. 68-70, Feb. 2001.

[2] J. A. Rubino-Martin et al., "The quijote CMB experiment," in Proc. 8th Sci. Meet. Spanish Astron. Soc., Santander, Spain, Jul. 2008, pp. 1-9. [Online]. Available: https://arxiv.org/abs/0810.3141

[3] E. Villa et al., "The thirty gigahertz instrument receiver for the Q-U-I joint tenerife experiment: Concept and experimental results," Rev. Sci. Instrum., vol. 86, no. 2, p. 024702, 2015.

[4] P. T. Timbie, J. O. Gundersen, and B. G. Keating, "Overview of cosmic microwave background polarization experiments," in Proc. ASP Conf. (AMiBA), vol. 257. 2002, pp. 235-242.

[5] E. Villa, J. Cagigas, B. Aja, L. de la Fuente, and E. Artal, "Q-band 4-state phase shifter in planar technology: Circuit design and performance analysis," Rev. Sci. Instrum., vol. 87, no. 9, p. 094705, 2016.

[6] X. Xu, R. G. Bosisio, and K. Wu, "A new six-port junction based on substrate integrated waveguide technology," IEEE Trans. Microw. Theory Techn., vol. 53, no. 7, pp. 2267-2273, Jul. 2005.

[7] E. Moldovan, R. G. Bosisio, and K. Wu, "W-band multiport substrateintegrated waveguide circuits," IEEE Trans. Microw. Theory Techn., vol. 54, no. 2, pp. 625-632, Feb. 2006.

[8] A. Doghri, T. Djerafi, A. Ghiotto, and K. Wu, "Broadband substrateintegrated-waveguide six-port applied to the development of polarimetric imaging radiometer," in Proc. 41st Eur. Microw. Conf. (EuMC), Manchester, U.K., Oct. 2011, pp. 393-396.

[9] J. Chen, W. Hong, P. Yan, B. Liu, Y. Wang, and K. Wu, "Design of a sixport junction using half-mode substrate integrated waveguide," in Proc. Asia-Pacific Microw. Conf., Bangkok, Thailand, Dec. 2007, pp. 1-4.

[10] Y. Ding and K. Wu, "Half-mode substrate integrated waveguide sixport front-end circuits for direct-conversion transceiver design," in IEEE MTT-S Int. Microw. Symp. Dig., Atlanta, GA, USA, Jun. 2008, pp. $1175-1178$.

[11] J. L. Cano, A. Mediavilla, and A. R. Perez, "Full-band air-filled waveguide-to-substrate integrated waveguide (SIW) direct transition," IEEE Microw. Wireless Compon. Lett., vol. 25, no. 2, pp. 79-81, Feb. 2015.

[12] L. T. Hildebrand, "Results for a simple compact narrow-wall directional coupler," IEEE Microw. Guided Wave Lett., vol. 10, no. 6, pp. 231-232, Jun. 2000.

[13] D. Deslandes and K. Wu, "Accurate modeling, wave mechanisms, and design considerations of a substrate integrated waveguide," IEEE Trans. Microw. Theory Techn., vol. 54, no. 6, pp. 2516-2526, Jun. 2006.

[14] J.-X. Chen, W. Hong, Z.-C. Hao, H. Li, and K. Wu, "Development of a low cost microwave mixer using a broad-band substrate integrated waveguide (SIW) coupler," IEEE Microw. Wireless Compon. Lett., vol. 16, no. 2, pp. 84-86, Feb. 2006.

[15] D. Arai, M. Zhang, K. Sakurai, J. Hirokawa, and M. Ando, "Obliquely arranged feed waveguide for alternating-phase fed single-layer slotted waveguide array," IEEE Trans. Antennas Propag., vol. 53, no. 2, pp. 549-600, Feb. 2005.

[16] Y. Cheng, W. Hong, and K. Wu, "Novel substrate integrated waveguide fixed phase shifter for 180-degree directional coupler," in IEEE MTT-S Int. Microw. Symp. Dig., Jun. 2007, pp. 189-192.

[17] Y. J. Cheng, W. Hong, and K. Wu, "Broadband self-compensating phase shifter combining delay line and equal-length unequal-width phaser," IEEE Trans. Microw. Theory Techn., vol. 58, no. 1, pp. 203-210, Jan. 2010.

[18] I. Boudreau, K. Wu, and D. Deslandes, "Broadband phase shifter using air holes in Substrate Integrated Waveguide," in IEEE MTT-S Int. Microw. Symp. Dig., Baltimore, MD, USA, Jun. 2011, pp. 1-4.

[19] T. Djerafi, K. Wu, and S. O. Tatu, "Substrate-integrated waveguide phase shifter with rod-loaded artificial dielectric slab," Electron. Lett., vol. 51, no. 9, pp. 707-709, Apr. 2015 
[20] S. Germain, D. Deslandes, and K. Wu, "Development of substrate integrated waveguide power dividers," in Proc. IEEE Can. Conf. Elect. Comput. Eng. (CCECE), Montreal, QC, Canada, May 2003, pp. 1921-1924.

[21] J. Dittloff, J. Bornemann, and F. Arndt, "Computer aided design of optimum E- or H-plane N-furcated waveguide power dividers," in Proc. 17th Eur. Microw. Conf., Rome, Italy, Sep. 1987, pp. 181-186.

[22] A. Casanueva, J. A. Pereda, and A. Mediavilla, "Optimum compact H- and E-plane corners in rectangular waveguide," Microw. Opt. Technol. Lett., vol. 42, no. 6, pp. 494-497, Sep. 2004.

[23] D. Deslandes and K. Wu, "Design consideration and performance analysis of substrate integrated waveguide components," in Proc. 32nd Eur. Microw. Conf., Milan, Italy, Sep. 2002, pp. 1-4.

[24] J. L. Cano, B. Aja, E. Villa, L. de la Fuente, and E. Artal, "Broadband back-end module for radio-astronomy applications in the $\mathrm{Ka}$ band," in Proc. 38th Eur. Microw. Conf., Amsterdam, The Netherlands, Oct. 2008, pp. 1113-1116.

[25] E. Villa, B. Aja, L. de la Fuente, and E. Artal, "Performance evaluation of a lossy transmission lines based diode detector at cryogenic temperature," Rev. Sci. Instrum., vol. 87, no. 1, p. 014706, 2016.

[26] M. Bozzi, M. Pasian, L. Perregrini, and K. Wu, "On the losses in substrate-integrated waveguides and cavities," Int. J. Microw. Wireless Technol., vol. 1, no. 5, pp. 395-401, 2009.

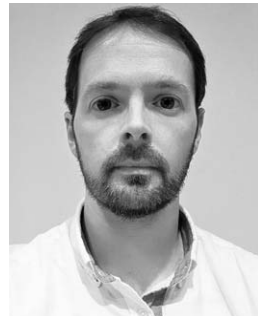

Juan Luis Cano was born in Torrelavega, Spain in 1979. He received the Telecommunications Engineering and $\mathrm{Ph} . \mathrm{D}$. degrees from the University of Cantabria, Santander, Spain, in 2004 and 2010, respectively.

$\mathrm{He}$ is currently a Researcher with the University of Cantabria, where he collaborates in the development and measurement of microwave receivers for radio astronomy and satellite applications. His current research interests include design and testing of lownoise amplifiers in MIC and monolithic microwave integrated circuit technologies both at room and cryogenic temperatures, design of different hardware for feed networks, and the development of new technologies for efficient subsystems in multipixel microwave cameras.

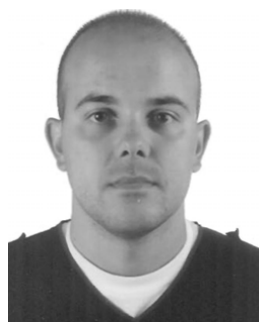

Enrique Villa was born in Santander, Spain. He received the Telecommunications Engineering and $\mathrm{Ph} . \mathrm{D}$. degrees from the University of Cantabria, Santander, in 2005 and 2014, respectively.

$\mathrm{He}$ is currently a Researcher with the University of Cantabria, where he collaborates in the design of low-noise, high-sensitivity, and broadband receivers for polarimetry. His current research interests include the design of phase switches both in MIC and in monolithic microwave integrated circuit technologies, the design of Schottky diodebased detectors, the design of receivers for radio astronomy, and the study and modeling of discrete devices both at room and cryogenic temperatures.

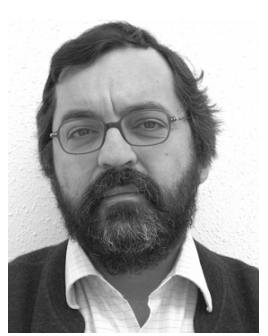

Angel Mediavilla was born in Santander, Spain, in 1955. He received the bachelor's degree and Ph.D degree (Hons.) in physics (electronic) from the University of Cantabria, Santander, Spain, in 1978 and 1983 , respectively.

From 1980 to 1983, he was an Ingenieur Stagiere with THOMSON-CSF. He has a wide experience in the analysis and optimization of nonlinear microwave active devices. He is currently a Full Professor with the Communications Engineering Department, University of Cantabria. His current research interests include nonlinear MESFET/HEMT and HBT device modeling with a special application to the large-signal computer design and new waveguide structures for antenna feed systems.

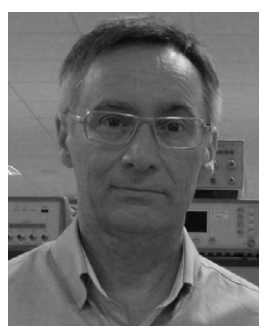

Eduardo Artal (M'81) received the Telecommunications Engineering and Ph.D. degrees from the Technical University of Catalonia, Barcelona, Spain, in 1976 and 1982, respectively.

From 1976 to 1990, he was an Assistant Professor with the Technical University of Catalonia. From 1979 to 1981, he was with Mier Allende S.A., Barcelona, where he was involved in TV and FM radio re-emitter development. Since 1990, he has been a Professor with the University of Cantabria, Santander, Spain. His current research interests include microwave circuits and systems and low-noise millimeterwave amplifiers and receivers. 